\title{
Group representations in indefinite metric spaces
}

\author{
P. M. van den Broek \\ Department of Applied Mathematics, Twente University of Technology, 7500 AE Enschede, The Netherlands
}

(Received 20 April 1983; accepted for publication 18 July 1983)

\begin{abstract}
A group $G$ of symmetry transformations of the rays of an indefinite metric space $V$ with metric operator $\eta$ leads to a projective representation $U$ of $G$ in $V$ in terms of $\eta$-unitary, $\eta$-antiunitary, $\eta$ pseudounitary, and $\eta$-pseudoantiunitary operators. We investigate the restrictions which are put on the irreducible components of $U$ by the metric, and examine to what extent it is possible to decompose $V$ into a direct sum of indefinite metric spaces, each carrying a projective representation of $G$. Attention is restricted to the cases where the subgroup of $G$ which is represented by $\eta$-unitary operators is of index 1 or 2 .
\end{abstract}

PACS numbers: $02.20 .+b$

\section{INTRODUCTION}

Our main motivation for studying group representations in indefinite metric spaces is the fact that twistor space is an indefinite metric space which carries in a natural way a (generalized) representation of the conformal group. ${ }^{1,2} \mathrm{How}$ ever, our results may be of interest in any field where indefinite metric spaces are used and symmetry arguments do play a role (Ref. 3 for references).

Let $V$ be a $n$-dimensional complex vector space, $n \geqslant 3$, with a scalar product denoted by (, ) and let $\eta$ be a Hermitian nonsingular linear operator on $V$. The indefinite metric of $V$ is given by

$$
\langle\phi, \psi\rangle=(\phi, \eta \psi),
$$

and $\eta$ is called the metric operator.

The rays of $V$ are the one-dimensional linear subspaces of $V$. If $\psi \in V$ and $\psi \neq 0$, then $\psi$ denotes the ray which contains $\psi$.

The rays $\psi$ and $\phi$ are said to be orthogonal (denoted by $\langle\psi \cdot \phi\rangle=0$ ) if and only if $\langle\psi, \phi\rangle=0$ for each $\psi \in \psi$ and each $\phi \in \phi$.

The rays of $V$ form the projective space $V$. Let $T$ be a bijective mapping of $\mathbf{V}$ onto $\mathbf{V}$ which has the property

$$
\langle\psi \cdot \phi\rangle=0 \Leftrightarrow\langle T \psi \cdot T \phi\rangle=0 .
$$

Then we have the following theorem:

Theorem 1: There exists a operator $U$ on $V$ such that

(i) $\psi \in \psi \Rightarrow U \psi \in T \psi$,

(ii) Either $U$ is linear and satisfies

$$
\langle U \phi, U \psi\rangle=\langle\phi, \psi\rangle \quad \forall \phi, \psi \in V,
$$

or $U$ is linear and satisfies

$$
\langle U \phi, U \psi\rangle=-\langle\phi, \psi\rangle \quad \forall \phi, \psi \in V,
$$

or $U$ is antilinear and satisfies

$$
\langle U \phi, U \psi\rangle=\langle\psi, \phi\rangle \quad \forall \phi, \psi \in V,
$$

or $U$ is antilinear and satisfies

$$
\langle U \phi, U \psi\rangle=-\langle\psi, \phi\rangle \quad \forall \phi, \psi \in V .
$$

Moreover, $U$ is determined uniquely by $T$ up to a factor of modulus 1 .

This theorem, first given in Ref. 4, is a generalization of a theorem in Ref. 3, which in turn is the generalization of Wigner's theorem to indefinite spaces. The set of operators enumerated under (ii) of the theorem are called $\eta$-unitary, $\eta$ pseudounitary, $\eta$-antiunitary, and $\eta$-pseudoantiunitary, respectively.

Let $G$ be a finite group and suppose that for each $g \in G$ there is a bijective mapping $T(g)$ of $V$ onto $V$ such that $E q$. (1.2) holds. Then the theorem above gives us for each $g \in G$ an operator $U(g)$ on $V$ which is either $\eta$-unitary, or $\eta$-antiunitary, or $\eta$-pseudounitary, or $\eta$-pseudoantiunitary. Since $U(g)$ is determined by $T(g)$ up to a factor of modulus 1 , we have

$$
U(g) U\left(g^{\prime}\right)=\sigma\left(g, g^{\prime}\right) U\left(g g^{\prime}\right) \quad \forall g, g^{\prime} \in G,
$$

where the mapping $\sigma$ of $G \times G$ into the complex numbers of modulus 1 is called a factor system of $G$.

Let $G_{0}$ be the normal subgroup of $G$ consisting of those elements $g$ of $G$ for which $U(g)$ is $\eta$-unitary. Let $a, b$, and $c$ be elements of $G$ (if any exist) such that $U(a)$ is $\eta$-antiunitary, $U(b)$ is $\eta$-pseudounitary, and $U(c)$ is $\eta$-pseudoantiunitary. Then $a G_{0}, b G_{0}$, and $c G_{0}$ denote the cosets of $G$ with respect to $G_{0}$ which consist of the elements $g$ of $G$ for which $U(g)$ is $\eta$ antiunitary, $\eta$-pseudounitary, and $\eta$-pseudoanitunitary, respectively. For the coset decomposition of $G$ with respect to $G_{0}$ there are the following five possibilities:
(I) $G=G_{0}$,
(II) $G=G_{0}+a G_{0}$
(III) $G=G_{0}+b G_{0}$,
(IV) $G=G_{0}+c G_{0}$
(V) $G=G_{0}+a G_{0}+b G_{0}+c G_{0}$.

From Eqs. (1.3)-(1.6), it follows that

$$
\langle U(g) \phi, U(g) \psi\rangle=(-)_{g}\langle\phi, \psi\rangle^{g},
$$

where $(-)_{g}$ is defined by

$$
(-)_{g}= \begin{cases}+1 & \text { if } g \in G_{0}+a G_{0} \\ -1 & \text { if } g \notin G_{0}+a G_{0}\end{cases}
$$

and, if $\lambda \in C, \lambda^{g}$ is defined by

$$
\lambda^{g}= \begin{cases}\lambda & \text { if } g \in G_{0}+b G_{0} \\ \lambda * & \text { if } g \notin G_{0}+b G_{0},\end{cases}
$$

the asterisk denoting complex conjugation.

Now choose a basis $\left\{e_{1}, e_{2}, \ldots, e_{n}\right\}$ of $V$ which is orthonormal with respect to the ordinary scalar product and form the matrices of $U(g)$ and $\eta$ via

$$
U(g) e_{i}=\sum_{j=1}^{n} U(g)_{j i} e_{j}
$$


and

$$
\eta e_{i}=\sum_{j=1}^{n} \eta_{j i} e_{j}
$$

These matrices are again denoted by $U(g)$ and $\eta$; they satisfy

$$
U(g) U\left(g^{\prime}\right)^{g}=\sigma\left(g, g^{\prime}\right) U\left(g g^{\prime}\right),
$$

where the upper index $g$ is defined as in Eq. (1.12), and

$$
U^{\dagger}(g) \eta U(g)=(-)_{g} \eta^{g}
$$

where $U^{\dagger}$ denotes the Hermitian conjugate of $U$.

Matrices $U(g)$ satisfying Eq. (1.15) form a projective linear-antilinear (PLA) representation of $G$. If all the matrices are unitary, they form a projective unitary-antiunitary (PUA) representation of $G$. For simplicity we call such representations PLA (resp. PUA) representations even if all operators are linear.

Two PLA representations $U$ and $U^{\prime}$ of $G$ are equivalent if there exists a nonsingular matrix $A$ such that

$$
U^{\prime}(g)=A^{-1} U(g) A^{g} \quad \forall g \in G .
$$

If $U$ satisfies Eq. (1.16), then $U^{\prime}$, defined by Eq. (1.17) for some nonsingular matrix $A$, satisfies

$$
U^{\prime \dagger}(g) \eta^{\prime} U^{\prime}(g)=(-)_{g} \eta^{\prime g},
$$

where

$$
\eta^{\prime}=A^{\dagger} \eta A
$$

So the equivalence transformation given by $A$ transforms the Hermitian matrix $\eta$ into the Hermitian matrix $\eta^{\prime}$, which has the same signature as $\eta$. $U$ is said to be decomposable if there exists an equivalent PLA representation $U^{\prime}$ such that

$$
U^{\prime}(g)=\left(\begin{array}{cc}
U_{i}^{\prime}(g) & 0 \\
0 & U_{2}^{\prime}(g)
\end{array}\right)
$$

and

$$
\eta^{\prime}=\left(\begin{array}{cc}
\eta_{1}^{\prime} & 0 \\
0 & \eta_{2}^{\prime}
\end{array}\right) .
$$

Equation (1.18) then becomes

$$
U_{i}^{\prime \dagger}(g) \eta_{i}^{\prime} U_{i}^{\prime}(g)=(-)_{g} \eta_{i}^{\prime g} \quad(i=1,2)
$$

The aim of this paper is twofold. Our first objective is to examine to what extent it is possible to decompose a PLA representation of $G$ which satisfies Eq. (1.16). A decomposable PLA representation of $G$ is reducible, but the reverse is not generally true, due to the restriction made in Eq. (1.21). Our second objective is to examine which restrictions are put on the irreducible components of $U$ by Eq. (1.16). We will restrict ourselves to the case where $G_{0}$ is a subgroup of index 1 or 2 of $G$; i.e., we consider the cases I, II, III, and IV of Eq. (1.9). The study of case $V$ is left to a later paper.

\section{GENERAL REDUCTION}

Due to a result of Murthy, $U$ is equivalent to a PLA representation whose matrices are all unitary, i.e., there exists a nonsingular matrix $A$ such that the matrices $U^{\prime}(g)$ given by

$$
U^{\prime}(g)=A^{-1} U(g) A^{g}
$$

form a PUA representation of $G$. Equation (1.16) then be- comes

$$
\eta^{\prime} U^{\prime}(g)=(-)_{g} U^{\prime}(g) \eta^{\prime g},
$$

where

$$
\eta^{\prime}=A^{\dagger} \eta A
$$

There exists a unitary matrix $X$ such that $\eta^{\prime \prime}$, defined by

$$
\eta^{\prime \prime}=X^{\dagger} \eta^{\prime} X
$$

is a real diagonal matrix. If

$$
U^{\prime \prime}(g)=X^{-1} U^{\prime}(g) X^{g},
$$

then $U^{\prime \prime}$ is a PUA representation of $G$ which satisfies

$$
\eta^{\prime \prime} U^{\prime \prime}(g)=(-)_{g} U^{\prime \prime}(g) \eta^{\prime \prime} \text {. }
$$

Writing out matrix elements gives

$$
\eta_{i i}^{\prime \prime} U^{\prime \prime}(g)_{i j}=(-)_{g} U^{\prime \prime}(g)_{i j} \eta_{j j}^{\prime \prime} \text {. }
$$

Now consider the cases I and II [Eqs. (1.9a) and (1.9b)]. In these cases $(-)_{g}=+1$ for each $g \in G$. Then $U^{\prime \prime}(g)_{i j}=0$ whenever $\eta_{i i}^{\prime \prime} \neq \eta_{j j}^{\prime \prime}$. So by a suitable renumbering of rows and columns we have

$$
U^{\prime \prime}(g)=\sum_{i} \oplus U_{i}^{\prime \prime}(g)
$$

and

$$
\eta^{\prime \prime}=\sum_{i} \oplus \eta_{i i}^{\prime \prime}
$$

where each $\eta_{i}^{\prime \prime}$ is a multiple of the unit matrix.

So we have decomposed $U^{\prime \prime}$ as a direct sum of PUA representations $U_{i}^{\prime \prime}$ of $G$ and $\eta^{\prime \prime}$ in a corresponding direct sum of real multiples of the unit matrix. Each $U_{i}^{\prime \prime}$ can now be reduced to a direct sum of irreducible PUA representations of $G$, and this equivalence transformation does not change $\eta_{i}^{\prime \prime}$. So in the cases I and II our goal has been achieved; we have proved the following theorem.

Theorem 2: In the cases I and II there are no restrictions put by the metric on the irreducible components of a PLA representation $U$ of $G$, and $U$ is decomposable into irreducible PUA representations.

Now consider the cases where $g \in G$ exist with $(-)_{g}$ $=-1$. Then we have from Eq. (2.7) that $U^{\prime \prime}(g)_{i j}=0$ whenever $\left|\eta_{i i}^{\prime \prime}\right| \neq\left|\eta_{j j}^{\prime \prime}\right|$. So by a suitable renumbering of rows and columns we have the decomposition of Eqs. (2.8) and (2.9); but here each $\eta_{i}^{\prime \prime}$ is a real diagonal matrix with all diagonal elements equal up to a sign.

Each $U_{i}^{\prime \prime}$ can now be studied separately; it satisfies

$$
\eta_{i}^{\prime \prime} U_{i}^{\prime \prime}(\mathrm{g})=(-)_{g} U_{i}^{\prime \prime}(g) \eta_{i}^{\prime \prime} \text {. }
$$

Let the diagonal elements of $\eta_{i}^{\prime \prime}$ be $a_{i}$ and $-a_{i}$ with $a_{i}>0$. Since $g \in G$ exists with $(-)_{g}=-1$, it follows from Eq. (2.10) that $\operatorname{Tr} \eta_{i}^{\prime \prime}=0$. So $a_{i}$ and $-a_{i}$ have equal multiplicity.

The equivalence transformation on $U_{i}^{\prime \prime}$ and $\eta_{i}^{\prime \prime}$ by the matrix $A_{i}$ which is equal to $a_{i}^{-1 / 2}$ times the unit matrix leaves $U_{i}^{\prime \prime}$ unchanged, but turns $\eta_{i}^{\prime \prime}$ into a diagonal matrix with diagonal elements +1 and -1 with equal multiplicity. So, without loss of generality, we may assume that $\eta_{i}^{\prime \prime}$ has eigenvalues +1 and -1 . In the next section we study case III and in Sec. 4 we treat case IV. 


\section{3. $\eta$-UNITARY AND $\eta$-PSEUDOUNITARY OPERATORS}

In this section we consider case III [Eq. (1.9c)], i.e.,, the case where $G$ is represented by $\eta$-unitary and $\eta$-pseudounitary operators. $G_{0}$, the subgroup of $G$ which is represented by $\eta$-unitary operators, has index 2 . According to the theory of induced PUA representations, which in its most general form is given in Ref. 6, each irreducible PUA representation of $G$ is either of type A or of type B.

A PUA representation of type $A$ is equivalent to a PUA representation $D$, which can be written as

$$
\begin{aligned}
& D(g)=\left(\begin{array}{cc}
\Delta(g) & 0 \\
0 & \tilde{\Delta}(g)
\end{array}\right) \quad \forall g \in G_{0}, \\
& D(b)=\left(\begin{array}{cc}
0 & \sigma(b, b) \Delta\left(b^{2}\right) \\
\mathbf{1}_{d} & 0
\end{array}\right) .
\end{aligned}
$$

Here $\Delta$ is an irreducible PUA representation of $G_{0} ; \tilde{\Delta}$ is an irreducible PUA representation of $G_{0}$ which is related to $\Delta$ by

$$
\tilde{\Delta}(g)=\sigma(g, b) \sigma^{*}\left(b, b^{-1} g b\right) \Delta\left(b^{-1} g b\right)
$$

and which is not equivalent to $\Delta$. The dimension of $\Delta$ is $d$. $\mathbf{1}_{d}$ denotes the unit matrix of dimension $d . D$ is determined up to equivalence by the equivalence classes of PUA representations of $G_{0}$ containing $\Delta$ and $\tilde{\Delta}$. A PUA representation $D$ of type $\mathrm{B}$ has the property that its restriction $\Delta=D \downarrow G_{0}$ to $G_{0}$ is irreducible. In this case $\Delta$ and $\tilde{\Delta}$ are equivalent. $D$ is not uniquely determined by $\Delta$ : The PUA representation $D^{\prime}$ of $G$, given by

$$
D^{\prime}(g)= \begin{cases}D(g) & \text { if } g \in G_{0} \\ -D(g) & \text { if } g \notin G_{0},\end{cases}
$$

has the same restriction to $G_{0}$, but is not equivalent to $D$. However, the pair $\left(D, D^{\prime}\right)$ is determined up to equivalence by the equivalence class of $\Delta$. A pair of PUA representations of $G$ of type B which are not equivalent, but whose restrictions to $G_{0}$ are equivalent, are said to be related. Let $U$ be a PUA representation of $G$ with

$$
\eta U(g)=(-)_{g} U(g) \eta
$$

Due to the results of the previous section, we may assume that the Hermitian matrix $\eta$ has eigenvalues +1 and -1 only, with equal multiplicity. We may now perform a unitary equivalence transformation

$$
\begin{aligned}
& U^{\prime}(g)=W^{-1} U(g) W, \\
& \eta^{\prime}=W^{\dagger} \eta W
\end{aligned}
$$

such that the PUA representation $U^{\prime}$ of $G$ has the following properties:

(i) $U^{\prime}$ is a direct sum of irreducible PUA representations $D_{i}$.

(ii) The components $D_{i}$ of this direct sum are pairwise either equal or inequivalent.

(iii) Components of type A have the form of Eqs. (3.1) and (3.2). (3.4).

(iv) Components of type B which are related satisfy Eq.

(v) The components are arranged into blocks $U_{i}^{\prime}$ :

$$
U^{\prime}(g)=\sum_{i} \oplus U_{i}^{\prime}(g)
$$

according to the following rules:

(1) irreducible components of type $A$ are in the same block if and only if they are equivalent (and thus equal);

(2) irreducible components of type B are in the same block if and only if they are either equal or related.

Note that $\eta$ can be chosen to be a diagonal matrix due to the results of the previous section, but this property is not inherited by $\eta^{\prime}$. If the Hermitian matrix $\eta^{\prime}$ is divided into blocks in the same way as $U^{\prime}$,

$$
\eta^{\prime}=\left(\begin{array}{ll}
\eta_{[1,1]}^{\prime} \cdots \eta_{[1, p]}^{\prime} \\
\eta_{[p, 1]}^{\prime} \cdots \eta_{[p, p]}^{\prime}
\end{array}\right),
$$

we find

$$
\eta^{\prime}{ }_{[i, k]} U_{k}^{\prime}(g)=(-)_{g} U_{i}^{\prime}(g) \eta_{[i, k]}^{\prime} .
$$

If $i \neq k$, the blocks $U_{k}^{\prime}$ and $U_{i}^{\prime}$, when restricted to $G_{0}$, are PUA representations of $G_{0}$ which have no irreducible components in common. Due to Schur's lemma, we thus have

$$
\eta_{[i, k]}^{\prime}=0
$$

if $i \neq k$. This means that we have found a decomposition of $U^{\prime}$ into blocks $U_{i}^{\prime}$; each block $U_{i}^{\prime}$ can now be studied further separately. Let $V$ be some block $U_{i}^{\prime}$, and let $\xi$ be the Hermitian matrix $\eta_{[i . i]}^{\prime}$. Then

$$
\xi V(g)=(-)_{g} V(g) \zeta .
$$

$\zeta$ is Hermitian and has eigenvalues +1 and -1 with equal multiplicity. Consider first the case that $V$ consists of irreducible PUA representations $D$ of $G$ type A. We may then write

$$
\begin{aligned}
& V(g)=\mathbb{1}_{n} \otimes\left(\begin{array}{cc}
\Delta(g) & 0 \\
0 & \tilde{\Delta}(g)
\end{array}\right) \quad \forall g \in G_{0}, \\
& V(b)=\mathbb{1}_{n} \otimes\left(\begin{array}{cc}
0 & \sigma(b, b) \Delta\left(b^{2}\right) \\
1_{d} & 0
\end{array}\right) .
\end{aligned}
$$

Here $n$ is the multiplicity of $D$ in $V$. Divide $\xi$ into blocks in the same way as $V$ :

$$
\xi=\left(\begin{array}{c}
\zeta_{[1,1]} \xi_{\{1,2]} \cdots \xi_{[1, n]} \\
\zeta_{[n, 1]} \cdots \zeta_{[n, n]}
\end{array}\right)
$$

From Eqs. (3.12)-(3.14) and Schur's lemma it follows that each $\xi_{[i j]}$ satisfies

$$
\zeta_{[i j]}=\left(\begin{array}{cc}
a_{i j} \mathbf{1}_{d} & 0 \\
0 & -a_{i j} \mathbb{1}_{d}
\end{array}\right)
$$

for some $a_{i j} \in C$. So we can write

$$
\zeta=A \otimes\left(\begin{array}{cc}
\mathbb{1}_{d} & 0 \\
0 & -\mathbb{1}_{d}
\end{array}\right),
$$

where $A$ is a Hermitian $n \times n$ matrix. There exists a unitary matrix $X$ such that $A^{\prime}$, defined by

$$
A^{\prime}=X^{\dagger} A X
$$

is a diagonal matrix; its diagonal elements are equal to +1 or -1 . Define the unitary matrix $Y$ by

$$
Y=X \otimes\left(\begin{array}{cc}
\mathbb{1}_{d} & 0 \\
0 & \mathbb{1}_{d}
\end{array}\right)
$$

and perform the equivalence transformation on $V$ and $\zeta$ by 
$Y$. Then $V$ is left unchanged, but $\zeta$ turns into $\zeta^{\prime}$, where

$$
\zeta^{\prime}=A^{\prime} \otimes\left(\begin{array}{cc}
\mathbf{1}_{d} & 0 \\
0 & -\mathbf{1}_{d}
\end{array}\right) \text {. }
$$

So $\zeta^{\prime}$ is a diagonal matrix with diagonal elements +1 and -1 with equal multiplicity. This means that $V$ has been decomposed to a direct sum of irreducible PUA representations of $G$.

Now consider the case that $V$ consists of irreducible PUA representations of $G$ of type B; suppose the multiplicity of $D$ in $V$ is $n$ and the multiplicity of $D^{\prime}$ in $V$ is $m$. With a suitable numbering of rows and columns we have

$$
V(g)=\left(\begin{array}{cc}
\mathbb{1}_{n} \otimes D(g) & 0 \\
0 & \mathbf{1}_{m} \otimes D(g)
\end{array}\right) \quad \forall g \in G_{0}
$$

and

$$
V(b)=\left(\begin{array}{cc}
\mathbf{1}_{n} \otimes D(b) & 0 \\
0 & -\mathbb{1}_{m} \otimes D(b)
\end{array}\right) .
$$

Let $\zeta$ be divided accordingly:

$$
\zeta=\left(\begin{array}{ll}
\zeta_{11} & \zeta_{12} \\
\zeta_{21} & \zeta_{22}
\end{array}\right)
$$

From Eqs. (3.12) and (3.21) and Schur's lemma it follows that $\zeta_{11}=0$ and $\xi_{22}=0$. Since det $\xi \neq 0$, the dimension of $\xi_{11}$ and $\zeta_{22}$ must be equal. Thus $n=m$, and we arrive at the following restriction put on the irreducible components of $U$ by the metric:

Theorem 3: In a PLA representation of $G$ belonging to case III related irreducible PUA representations have the same multiplicity.

From Eq. (3.12), Schur's lemma, and the Hermiticity of $\zeta$, it follows that

$$
\zeta_{12}=\chi \otimes \mathbb{1}_{d}
$$

and

$$
\zeta_{21}=\chi^{\dagger} \otimes 1_{d}
$$

for some $n \times n$ matrix $\chi$. Since $\zeta$ is Hermitian and has eigenvalues +1 and -1 only, the matrix $\zeta^{2}$ equals $1_{n}$. This implies that $\chi$ is unitary. Define the unitary matrix $Y$ by

$$
Y=\left(\begin{array}{cc}
\mathbb{1}_{n d} & 0 \\
0 & \chi^{\dagger} \otimes \mathbb{1}_{d}
\end{array}\right)
$$

and perform the equivalence transformation on $V$ and $\zeta$ by $Y$. Then $V$ is left unchanged, but $\xi$ turns into $\zeta^{\prime}$, where

$$
\zeta^{\prime}=\left(\begin{array}{cc}
0 & 1_{n d} \\
1_{n d} & 0
\end{array}\right) .
$$

By a suitable reordering of rows and columns, we may now write $V$ and $\xi^{\prime}$ as follows:

$$
\begin{aligned}
& V(g)=\mathbb{1}_{n} \otimes\left(\begin{array}{cc}
D(g) & 0 \\
0 & D(g)
\end{array}\right) \quad \forall g \in G_{0}, \\
& V(b)=\mathbb{1}_{n} \otimes\left(\begin{array}{cc}
D(b) & 0 \\
0 & -D(b)
\end{array}\right), \\
& \zeta^{\prime}=\mathbb{1}_{n} \otimes\left(\begin{array}{cc}
0 & 1_{d} \\
1_{d} & 0
\end{array}\right) .
\end{aligned}
$$

So $V$ has been decomposed into components which are pairs of an irreducible PUA representation and its related PUA representation. A further decomposition of these components is not possible. This follows from the fact that if $V$ is an irreducible PUA representation of $G$ of type B, there exists no Hermitian matrix $\zeta$ which satisfies Eq. (3.12). So we have derived the following theorem:

Theorem 4: Any PLA representation of $G$ belonging to case III is decomposable into irreducible PUA representations of type $A$ and pairs of related irreducible PUA representations of type $B$.

\section{4. $\eta$-UNITARY AND $\eta$-PSEUDOANTIUNITARY OPERATORS}

In this section we consider case IV [Eq. (1.9d)], i.e., the case where $G$ is represented by $\eta$-unitary and $\eta$-pseudoantiunitary operators. $G_{0}$, the subgroup of $G$ which is represented by $\eta$-unitary operators had index 2 . Each irreducible PUA representation of $G$ is either of type A, or of type B or of type C.

A PUA representation $D$ of type A has the property that its restriction $\Delta=D \downarrow G_{0}$ to $G_{0}$ is irreducible. $D$ is determined up to equivalence by the equivalence class of $\Delta$. A PUA representation of type $B$ is equivalent to a PUA representation $D$, which can be written as

$$
\begin{aligned}
& D(g)=\left(\begin{array}{cc}
\Delta(g) & 0 \\
0 & \Delta(g)
\end{array}\right) \quad \forall g \in G_{0}, \\
& D(c)=\left(\begin{array}{cc}
0 & \mathscr{D} \\
-\mathscr{D} & 0
\end{array}\right) .
\end{aligned}
$$

$D$ is determined up to equivalence by the equivalence class of $\Delta$. A PUA representation of type $C$ is equivalent to a PUA representation $D$, which can be written as

$$
\begin{aligned}
& D(g)=\left(\begin{array}{cc}
\Delta(g) & 0 \\
0 & \tilde{\Delta}(g)
\end{array}\right) \quad \forall g \in G_{0}, \\
& D(c)=\left(\begin{array}{cc}
0 & \sigma(c, c) \Delta\left(c^{2}\right) \\
\mathbb{1}_{d} & 0
\end{array}\right) .
\end{aligned}
$$

$\Delta$ and $\tilde{\Delta}$ are irreducible $d$-dimensional PUA representations of $G_{0}$, which are not equivalent to each other and are related by

$$
\tilde{\Delta}(g)=\sigma(g, c) \sigma^{*}\left(c, c^{-1} g c\right) \Delta{ }^{*}\left(c^{-1} g c\right) \text {. }
$$

$D$ is determined up to equivalence by the equivalence classes of $\Delta$ and $\tilde{\Delta}$. Let $U$ be a PUA representation of $G$ with

$$
\eta U(g)=(-)_{g} U(g) \eta^{g} .
$$

Due to the results of Sec. 2, we may assume that the Hermitian matrix $\eta$ has eigenvalues +1 and -1 only, with equal multiplicity. We may now perform a unitary equivalence transformation

$$
\begin{aligned}
& U^{\prime}(g)=W^{-1} U(g) W^{8}, \\
& \eta^{\prime}=W^{\dagger} \eta W
\end{aligned}
$$

such that the PUA representation $U^{\prime}$ of $G$ has the following properties:

(i) $U^{\prime}$ is a direct sum of irreducible PUA representations $D_{i}$.

(ii) The components $D_{i}$ of this direct sum are pairwise either equal or inequivalent.

(iii) Components of type B have the form of Eqs. (4.1) 
and (4.2).

(iv) Components of type $\mathrm{C}$ have the form of Eqs. (4.3) and (4.4).

(v) The components are arranged into blocks $U_{i}^{\prime}$ :

$$
U^{\prime}(g)=\sum_{i} \oplus U_{i}^{\prime}(g)
$$

such that irreducible components of $U^{\prime}$ are in the same block if and only if they are equivalent (and thus equal).

Equation (4.9) is a decomposition of $U^{\prime}$. This is proved in the same way as the corresponding decomposition in the previous section. So each block $U_{i}^{\prime}$ can now be studied separately.

Let $V$ be some block $U_{i}^{\prime}$, and let $\xi$ be the corresponding block of $\eta^{\prime}$. Then

$$
\zeta V(g)=(-)_{g} V(g) \zeta^{g} .
$$

$\zeta$ is Hermitian and has eigenvalues +1 and -1 with equal multiplicity. Consider first the case that $V$ consists of irreducible PUA representations $D$ of $G$ of type A. Then we may write

$$
V(g)=\mathbb{1}_{n} \otimes \Delta(g) \quad \forall g \in G_{0}
$$

and

$$
V(c)=\mathbb{I}_{n} \otimes D(c) .
$$

Here $n$ is the multiplicity of $D$ in $V$. Divide $\xi$ into blocks in the same way as $V$ [Eq. (3.15)]. Then it follows from Eqs. (4.10) and (4.11) and Schur's lemma that

$$
\xi_{[i, j]}=a_{i j} 1_{d}
$$

for some $a_{i j} \in C$. We thus have

$$
\boldsymbol{\zeta}=A \otimes \mathbf{1}_{d}
$$

for some Hermitian $n \times n$ matrix $A$. From Eqs. (4.10) and (4.12) it now follows that

$$
A=-A^{*} \text {. }
$$

Thus the dimension of $A$ is even, and thus we have arrived at the following restriction put by the metric on the irreducible components of $U$ :

Theorem 5: In a PLA representation of $G$ belonging to case IV the irreducible PUA representations of type A have even multiplicity.

Now there exists an orthogonal matrix $B$ such that

$$
B^{\dagger} A B=\mathbb{1}_{n / 2} \otimes\left(\begin{array}{cc}
0 & i \\
-i & 0
\end{array}\right) \text {. }
$$

This follows from $\$ 13$ of Chap. IX of Ref. 7, since $i A$ is real, skew-symmetric, and has eigenvalues $+i$ and $-i$ with equal multiplicity. Define

$$
C=B \otimes \mathbb{1}_{d}
$$

and perform the equivalence transformation on $V$ and $\zeta$ by $C$. Then $V$ is left unchanged, and $\zeta$ is transformed into

$$
\zeta^{\prime}=\mathbb{1}_{q / 2} \otimes\left(\begin{array}{cc}
0 & i \mathbb{1}_{d} \\
-i \mathbb{1}_{d} & 0
\end{array}\right) \text {. }
$$

It is seen that we have decomposed $V$ into blocks containing two equal irreducible PUA representations of type $\mathbf{A}$.

Further decomposition is not possible, due to Theorem 5.

Next consider the case where $V$ consists of irreducible
PUA representations $D$ of type $B$. With a suitable numbering of rows and columns, we may write

$$
V(g)=\left(\begin{array}{cc}
\mathbb{1}_{n} \otimes \Delta(g) & 0 \\
0 & \mathbb{1}_{n} \otimes \Delta(g)
\end{array}\right) \quad \forall g \in G_{0}
$$

and

$$
V(c)=\left(\begin{array}{cc}
0 & \mathbb{1}_{n} \otimes \mathscr{D} \\
-\mathbb{1}_{n} \otimes \mathscr{D} & 0
\end{array}\right) .
$$

From Eqs. (4.10), (4.19), and (4.20) and Schur's lemma it follows that

$$
\zeta=\chi \otimes 1_{d},
$$

where $\chi$ is a Hermitian $2 n \times 2 n$ matrix which is partitioned into square blocks as follows:

$$
\chi=\left(\begin{array}{cc}
\chi_{1} & \chi_{2} \\
\chi_{2}^{*} & -\chi_{1}^{*}
\end{array}\right) .
$$

Due to a lemma, which we prove in the Appendix, there exists a unitary $2 n \times 2 n$ matrix $U$ which is partitioned into square blocks as follows:

$$
U=\left(\begin{array}{cc}
U_{1} & U_{2} \\
-U_{2}^{*} & U_{1}^{*}
\end{array}\right)
$$

and which satisfies

$$
U^{\dagger} \chi U=\left(\begin{array}{cc}
\mathbb{1}_{n} & 0 \\
0 & -\mathbb{1}_{n}
\end{array}\right) .
$$

Define the unitary matrix $Y$ by

$$
Y=U \otimes \mathbf{1}_{d}
$$

and perform the equivalence transformation on $V$ and $\xi$ by $Y$. Then $V$ is left unchanged and $\zeta$ is transformed into

$$
\zeta^{\prime}=U^{\dagger} \chi U \otimes \mathbb{1}_{d}=\left(\begin{array}{cc}
\mathbb{1}_{n d} & 0 \\
0 & -\mathbb{1}_{n d}
\end{array}\right) .
$$

This means that $V$ has been decomposed to a direct sum of irreducible PUA representations of $G$.

Finally consider the case that $V$ consists of irreducible PUA representations $D$ of type $C$. With a suitable numbering of rows and columns, we may write

$$
V(g)=\left(\begin{array}{cc}
\mathbf{1}_{n} \otimes \Delta(g) & 0 \\
0 & \mathbf{1}_{n} \otimes \tilde{\Delta}(g)
\end{array}\right) \quad \forall g \in G_{0}
$$

and

$$
V(c)=\left(\begin{array}{cc}
0 & \sigma(c, c) \mathbf{1}_{n} \otimes \Delta\left(c^{2}\right) \\
\mathbf{1}_{n d} & 0
\end{array}\right) .
$$

From Eqs. (4.10), (4.27), and (4.28) and Schur's lemma it follows that

$$
\xi=\chi \otimes \mathbb{1}_{d},
$$

where $\chi$ is a Hermitian $2 n \times 2 n$ matrix which is partitioned into square blocks as follows:

$$
\chi=\left(\begin{array}{cc}
\chi_{1} & 0 \\
0 & -\chi_{1}^{*}
\end{array}\right) .
$$

Let $U$ be a unitary $n \times n$ matrix such that $U^{\dagger} \chi_{1} U$ is on diagonal form. Define the unitary matrix $Y$ by

$$
Y=\left(\begin{array}{cc}
U & 0 \\
0 & U^{*}
\end{array}\right) \otimes \mathbb{I}_{d}
$$


and perform the equivalence transformation on $V$ and $\zeta$ by $Y$. Then $V$ is left unchanged and $\zeta$ is transformed into

$$
\zeta^{\prime}=\left(\begin{array}{cc}
U^{\dagger} \chi_{1} U & 0 \\
0 & -\left(U^{\dagger} \chi_{1} U\right)^{*}
\end{array}\right) \otimes \mathbb{1}_{d},
$$

which is on diagonal form. This means that $V$ has been decomposed into a direct sum of irreducible PUA representations of $G$. So we have derived the following theorem:

Theorem 6: Any PLA representation of $G$ belonging to case IV is decomposable into a direct sum of irreducible PUA representations of type $B$, irreducible PUA representations of type $C$, and pairs of irreducible PUA representations of type A.

\section{APPENDIX} lemma:

This appendix is devoted to the proof of the following

Lemma: Let $H$ be a $2 n \times 2 n$ Hermitian matrix which is partitioned into square blocks as follows:

$$
H=\left(\begin{array}{cc}
H_{1} & H_{2} \\
H_{2}^{*} & -H_{1}^{*}
\end{array}\right)
$$

and which has only eigenvalues +1 and -1 . Let $I$ be defined by

$$
I=\left(\begin{array}{cc}
\mathbb{1}_{n} & 0 \\
0 & -\mathbb{1}_{n}
\end{array}\right) .
$$

There exists a unitary matrix $U$ which is partitioned into square blocks as follows:

$$
U=\left(\begin{array}{cc}
U_{1} & -U_{2}^{*} \\
U_{2} & U_{1}^{*}
\end{array}\right) .
$$

and which has the property that

$$
U^{\dagger} H U=I \text {. }
$$

Proof: Since $\operatorname{Tr} H=0$ the eigenvalues +1 and -1 have equal multiplicity. Thus there exists a unitary matrix $V$ such that

$$
V^{\dagger} H V=I \text {. }
$$

This implies that

$$
H V=V I \text {. }
$$

If $V$ is partitioned into square blocks as follows,

$$
V=\left(\begin{array}{ll}
V_{1} & V_{3} \\
V_{2} & V_{4}
\end{array}\right)
$$

then Eq. (A6) can be written as

$$
\begin{aligned}
& H_{1} V_{1}+H_{2} V_{2}=V_{1}, \\
& H_{1} V_{3}+H_{2} V_{4}=-V_{3}, \\
& H_{2}^{*} V_{1}-H_{1}^{*} V_{2}=V_{2}, \\
& H_{2} V_{3}^{*}-H_{1}^{*} V_{4}=-V_{4} .
\end{aligned}
$$

Now define the matrix $U$ by

$$
U=\left(\begin{array}{ll}
U_{1} & U_{3} \\
U_{2} & U_{4}
\end{array}\right)=\left(\begin{array}{cc}
V_{1} & -V_{2}^{*} \\
V_{2} & V_{1}^{*}
\end{array}\right) .
$$

Then $U$ satisfies equations (A8)-(A11) if $V_{i}$ in these equations is replaced by $U_{i}$. Consequently,

$$
H U=U I \text {. }
$$

Since $U$ has the form prescribed by Eq. (A3), the lemma is proved if we show that $U$ is unitary. Since $V$ is unitary, we have

$$
V_{1}^{\dagger} V_{1}+V_{2}^{+} V_{2}=1
$$

Expressing $U^{\dagger} U$ in terms of $V_{1}$ and $V_{2}$ gives, with Eq. (A14),

$$
U^{\dagger} U=\left(\begin{array}{cc}
1 & -V_{1}^{\dagger} V_{2}^{*}+V_{2}^{\dagger} V_{1}^{*} \\
-V_{2}^{* \dagger} V_{1}+V_{1}^{* \dagger} V_{2} & \mathbb{1}
\end{array}\right) .
$$

Equation (A13) implies

$$
U^{\dagger} H U=U^{\dagger} U I \text {. }
$$

Taking the Hermitian adjoint of equation (A13) and multiplying with $U$ from the right gives

$$
U^{\dagger} H U=I U^{\dagger} U \text {. }
$$

From Eqs. (A16) and (A17), it follows that $U^{\dagger} U$ commutes with $I$. This fact, together with Eq. (A15), implies that $U^{\dagger} U$ is the unit matrix. Thus $U$ is unitary, which proves the lemma.

${ }^{\text {I}}$ R. Penrose, "Twistor theory, its aims and achievements," in Quantum Gravity, edited by C. J. Isham, R. Penrose, and D. W. Sciama (Clarendon, Oxford, 1975)

${ }^{2}$ P. M. van den Broek, "Twistor space and the conformal group," Memorandum No. 361, Department of Applied Mathematics, Twente University of Technology, The Netherlands, 1981.

${ }^{3}$ L. Bracci, G. Morchio, and F. Strocchi, Commun. Math. Phys. 41, 289-99 (1975).

${ }^{4}$ P. M. van den Broek, "Twistor geometry," Memorandum No. 410, Department of Applied Mathematics, Twente University of Technology, The Netherlands, 1982.

${ }^{5}$ V. Murthy, J. Math. Phys. 7, 853-7 (1966).

${ }^{6} R$. Shaw and J. Lever, Commun. Math. Phys. 38, 257-77 (1974).

${ }^{7}$ F. R. Gantmacher, The Theory of Matrices (Chelsea, New York, 1959), Vol. I. 